\title{
Reliability of causes of death in persons with Type I diabetes
}

\author{
I. Mühlhauser ${ }^{1,2}$, P. T. Sawicki ${ }^{1}$, M. Blank ${ }^{1}$, H. Overmann ${ }^{1}$, B. Richter ${ }^{1}$, M. Berger ${ }^{1}$ \\ ${ }^{1}$ Department of Metabolic Diseases and Nutrition (WHO-Collaborating Centre for Diabetes, \\ Heinrich-Heine University Düsseldorf, Germany \\ ${ }^{2}$ Department of Health Sciences and Education, University of Hamburg, Germany
}

\section{Abstract}

Aims/hypothesis. To compare causes of death assessed by a clinical review committee, the information given on death certificates, and ICD-codes provided by the State Documentation Office in deceased persons with Type 1 (insulin-dependent) diabetes mellitus.

Methods. A cohort of 3674 patients were monitored for $10 \pm 3$ (mean $\pm \mathrm{SD}$ ) years. Vital status and endstage diabetic complications were documented for 97\%; 251 patients had died. Causes of death were assessed by a clinical review committee and compared to the information provided by death certificates and ICD-9 codes.

Results. The review committee defined a leading cause of death in $94 \%$ of cases, whereas death certificates were available for $73 \%$ and ICD-codes for $79 \%$ of patients; $10 \%$ of death certificates could not be evaluated due to insufficient information. Diabetes was mentioned on $71 \%$ of death certificates, and renal dis- ease in $75 \%$ of cases with renal replacement therapy. There was acceptable agreement between the committee, death certificates and ICD-codes only for deaths due to neoplasma, and between the committee and death certificates for deaths due to acute myocardial infarction, cerebrovascular events and accidents. In only one out of four deaths due to hypoglycaemia and in four of seven deaths due to ketoacidosis was this diagnosis mentioned on the death certificate. No death due to hypoglycaemia or ketoacidosis and $41 \%$ due to suicide were identifiable by ICD-codes.

Conclusion/interpretation. Reliance on death certificates or ICD-codes as the only sources of information on the cause of specific mortality does not provide data of sufficient reliability for evaluation of clinical outcome in Type I diabetes. [Diabetologia (2002) 45:1490-1497]

Keywords Type I diabetes, mortality, causes of death, death certificates, ICD codes.
Death and causes of death are important outcome measures for the care of persons with Type I (insulindependent) diabetes mellitus. Accurate ascertainment of the causes of death is essential but methods used to

Received: 19 April 2002 / Revised: 1 July 2002

Published online: 19 September 2002

(C) Springer-Verlag 2002

Corresponding author: Dr. I. Mühlhauser, Heinrich-Heine Universität Düsseldorf, Klinik für Stoffwechselkrankheiten und Ernährung, Moorenstraße 5, D-40225 Düsseldorf, Germany. E-mail: Ingrid_Muehlhauser@uni-hamburg.de

Abbreviations: ICD, International Classification of Diseases; DERI, Diabetes Epidemiology Research International Mortality Study; E-codes, external causes of death. evaluate the causes of death are of different reliability. The proportion of deaths examined by necropsy decreases in many countries and death certificates are used as a single or predominant source of information in many studies. However, death certificates are not reliable in persons with Type II (non-insulin-dependent) diabetes mellitus, particularly because diabetes is frequently not recorded $[1,2]$. Death certificates are also widely used in studies including persons with Type I diabetes $[3,4,5,6,7]$. Accuracy and reproducibility of information on death certificates have been shown to be poor and classification systems vary, therefore clinical review committees have been proposed as the most reliable method for the evaluation of causes of death [8]. In the Diabetes Epidemiology 
Research International Mortality Study (DERI) such an approach has been favoured to compare the cause of specific mortality in diabetic children and adolescents [9].

The aim of this study was to compare causes of death as assessed by a clinical review committee, death certificates and ICD-9 codes in adult patients with Type I diabetes.

\section{Subjects and methods}

All 3674 adults with Type I diabetes (ketosis-prone) who were treated with insulin before the age of 31 years and who participated in the 5-day treatment and teaching programme for intensification of insulin therapy at the diabetes centre of the Düsseldorf University Hospital between September 1978 and December 1994 or in one of nine co-operating general hospitals ( $n=590)$ during 1985 and 1986 [10] were re-examined between August 1996 and September 1998, after 10 \pm 3 (means \pm SD) years. The study was approved by the ethical committee of the University of Düsseldorf. The study cohort and procedures have been described in detail elsewhere $[11,12]$.

Causes of death were ascertained by a clinical review committee of two diabetologists, a senior medical student and a paramedical clinical diabetes investigator by using all available sources of information. These included, hospital records including outpatient records of the diabetes centre and other hospitals, data from clinical studies, physician and police records, contacting diabetologists, relatives, emergency physicians and family physicians on circumstances of death, and necropsy reports including medicolegal necropsy. Death certificates were not used except for 11 cases. The review committee assessed a leading cause of death based upon the following list of 11 possible causes according to the International Classification of Diseases (ICD-9):

1. Cardiac death (ICD-9: 390-459, 798), including acute myocardial infarction (death within 30 days after a critical ischaemic myocardial event, and/or acute rupture of myocardium at any time), heart failure (death due to congestive heart failure, without an acute ischaemic event during the last 30 days), sudden death (no potentially fatal or critical event or disease present at least one hour before death), or fatal arrhythmia at any time and unexpected death (no critical event or disease present at least 24 hours before death)

2. Cerebrovascular death (ICD-9: 430-434), including ischaemic cerebral events and intracranial haemorrhagic events.

3. Pulmonary death (ICD-9: 453, 490-496, 500-508), including pulmonary embolism, fatal obstructive and other pulmonary disease, but excluding neoplasms and pulmonary infections.

4. Gastrointestinal death (ICD-9: 530-537, 550-553, 560, $571,577,578$ ), including pancreatitis, liver cirrhosis, intestinal perforation and gastrointestinal bleeding, but excluding neoplasms and infections.

5. Renal death (ICD-9: 580-589), death due to uraemia of any cause including denial or cessation of dialysis treatment.

6. Diabetes treatment associated death (ICD-9: 271, 276), including hypoglycaemia, ketoacidosis, hyperosmolar coma and death within 4 weeks after pancreas transplantation. Metabolic death was assigned if supported by blood glucose measurement or by circumstantial evidence when other reasons could be ruled out.
7. Surgery associated death (ICD-9: E870-876), including inhospital death during or after a maximum of 1 week after surgery for a non-critical disease.

8. Infection caused death (ICD-9: 1-139, 320-326, 390-392, $422,480-487$ ), any septicaemia or fatal infection including diabetic foot syndrome, tuberculosis, pneumonia, meningitis, myocarditis and other infectious diseases.

9. Neoplasma (ICD-9: 140-239), any fatal malignant disease.

10. Violent death (ICD-9: E800-876, E880-999), including fatal accidents, suicide, violence and intoxication.

11. Unknown cause of death (ICD-9: 799).

After publication of the results on the causes of death, as assessed by the review committee in 2000 [11, 12], death certificates were made available by the municipal registries for 182 of the 251 deceased patients. Reasons for not obtaining death certificates were the following: 51 patients had died outof-state or more than 10 years ago (death certificates are stored only for 10 years) and for 18 patients data were not provided. All available death certificates were independently evaluated by two diabetologists, masked to any patient characteristic and blinded to the cause of death as defined by the review committee and ICD-9 codes, respectively. The full statements on the death certificates relating to cause of death were examined. This data set contained details of immediate/primary, contributory/antecedent, and underlying causes of death as well as information provided by police/district attorney reports in certain cases. A leading cause of death was defined based upon a classification list as used by the review committee. In the case of disagreement between the two raters, agreement was obtained by consensus.

In October 2001, ICD-9 codes and in March 2002, the corresponding E-codes were provided by the State Documentation Office for 199 of the 251 deceased patients. For 52 patients ICD codes could not be retrieved ( $n=40$ had died out-of-state). Death certificates are coded by trained nosologists at Public Health Offices of the State of North-Rhine Westfalia.

The following descriptive analyses and comparisons were carried out: (i) Documentation of diabetes on the death certificate; (ii) In case of renal replacement therapy, documentation of renal disease on the death certificate; (iii) Comparison of causes of death between the review committee and death certificates; (iv) Comparison of causes of death between the review committee and ICD-codes.

\section{Results}

Vital status could be documented for 3570 (97\%) of the 3674 patients; 251 (7\%) patients had died. Characteristics of these patients at the time of death are summarized in Table 1. Further details of the total study cohort have been published $[11,12]$.

Of the 251 patients 136 (54\%) had died in hospital, 108 outside hospital, for seven patients such information was not available. A total of 31 patients were known to have had an autopsy, 26 of these patients had died in hospital and were autopsied by consent, the remaining five necropsies were required by law; 29 autopsy reports were available for evaluation. Necropsy includes histological examination of major organs. The primary and most useful sources of information about the causes of death were hospital discharge reports for 114 cases $(45 \%)$, the last treating 
physician for 58 cases (23\%), relatives for 40 cases $(16 \%)$, death certificates in 11 cases (4\%), and autopsy reports in 16 cases $(6 \%)$.

Causes of death, as defined by the review committee for men and women combined, are summarized in Table 2. The clinical review committee defined a leading cause of death in 235 of the 251 (94\%) deceased patients. The retrieval rate of death certificates and ICD-codes was comparable across the various causes of death (Table 2, data not shown for ICD-codes).

Table 1. Characteristics of patients at time of death

\begin{tabular}{lc}
\hline & Total group $n=251$ \\
\hline Age (years) & $42 \pm 13(19-88)$ \\
Diabetes duration (years) & $26 \pm 12(<1-68)$ \\
Endstage diabetic complications & \\
Blindness & $10.5 \%$ \\
Renal replacement therapy & $34.0 \%$ \\
Amputations & $12.5 \%$ \\
At least one endstage complication & $42.0 \%$ \\
Location of death & \\
In hospital & $136(54.2 \%)$ \\
Outside hospital & $108(43.0 \%)$ \\
Unknown & $7(2.8 \%)$ \\
\hline
\end{tabular}

Table 2. Causes of death

\begin{tabular}{|c|c|c|}
\hline & $\begin{array}{l}\text { Total group } \\
n=251\end{array}$ & $\begin{array}{l}\text { Sub-group } \\
\text { with death } \\
\text { certificates } \\
n=182\end{array}$ \\
\hline Cardiac & $104(41.4 \%)$ & $78(43.1 \%)$ \\
\hline Acute myocardial infarction & $32(12.7 \%)$ & $23(12.7 \%)$ \\
\hline Heart failure & $10(4.0 \%)$ & $7(3.9 \%)$ \\
\hline Sudden death & $28(11.2 \%)$ & $22(12.1 \%)$ \\
\hline Unexpected death & $34(13.5 \%)$ & $27(14.9 \%)$ \\
\hline Cerebrovascular & $20(8.0 \%)$ & $14(7.7 \%)$ \\
\hline Ischaemic & $9(3.6 \%)$ & $5(2.8 \%)$ \\
\hline Haemorrhagic & $11(4.4 \%)$ & $8(4.4 \%)$ \\
\hline Violent & $36(14.3 \%)$ & $20(11.0 \%)$ \\
\hline Accident & $14(5.6 \%)$ & $8(4.4 \%)$ \\
\hline Suicide & $22(8.8 \%)$ & $12(6.6 \%)$ \\
\hline Diabetes treatment associated & $18(7.2 \%)$ & $13(7.2 \%)$ \\
\hline Hypoglycaemia & $7(2.8 \%)$ & $4(2.2 \%)$ \\
\hline Ketoacidosis / coma & $8(3.2 \%)$ & $7(3.9 \%)$ \\
\hline Pancreas transplantation & $3(1.2 \%)$ & $2(1.1 \%)$ \\
\hline Infection & $25(10.0 \%)$ & $18(9.9 \%)$ \\
\hline Surgery associated & $8(3.2 \%)$ & $7(3.9 \%)$ \\
\hline Renal & $4(1.6 \%)$ & $4(2.2 \%)$ \\
\hline Neoplasma & $16(6.4 \%)$ & $12(6.6 \%)$ \\
\hline Pulmonary & $3(1.2 \%)$ & $3(1.7 \%)$ \\
\hline Gastrointestinal & $1(0.4 \%)$ & $1(0.6 \%)$ \\
\hline Unknown & $16(6.4 \%)$ & $12(6.6 \%)$ \\
\hline
\end{tabular}

Means $\pm \mathrm{SD}($ range $)$

Table 3. Comparison of the leading cause of death as defined by the clinical review committee and diagnoses mentioned on the corresponding death certificate

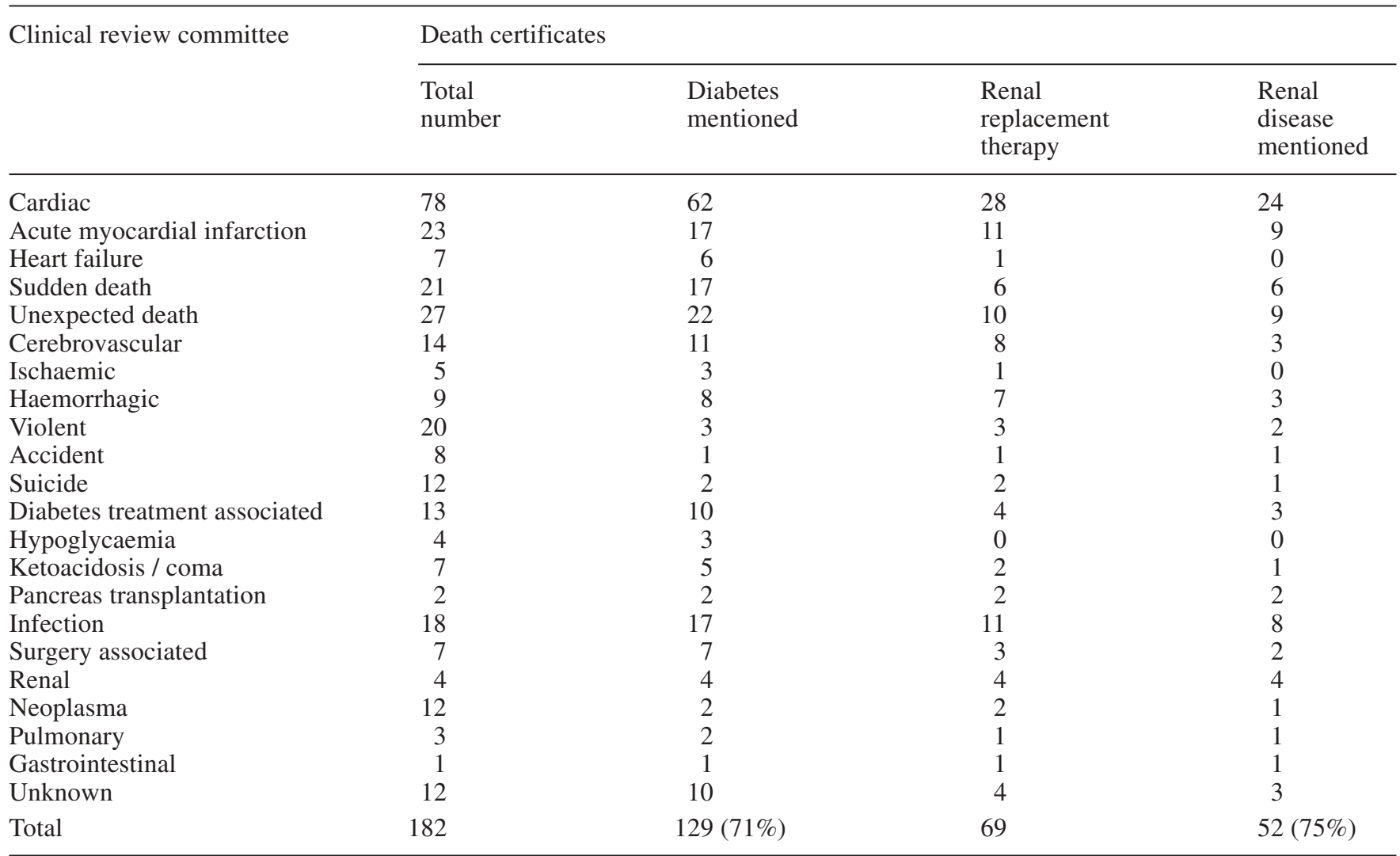




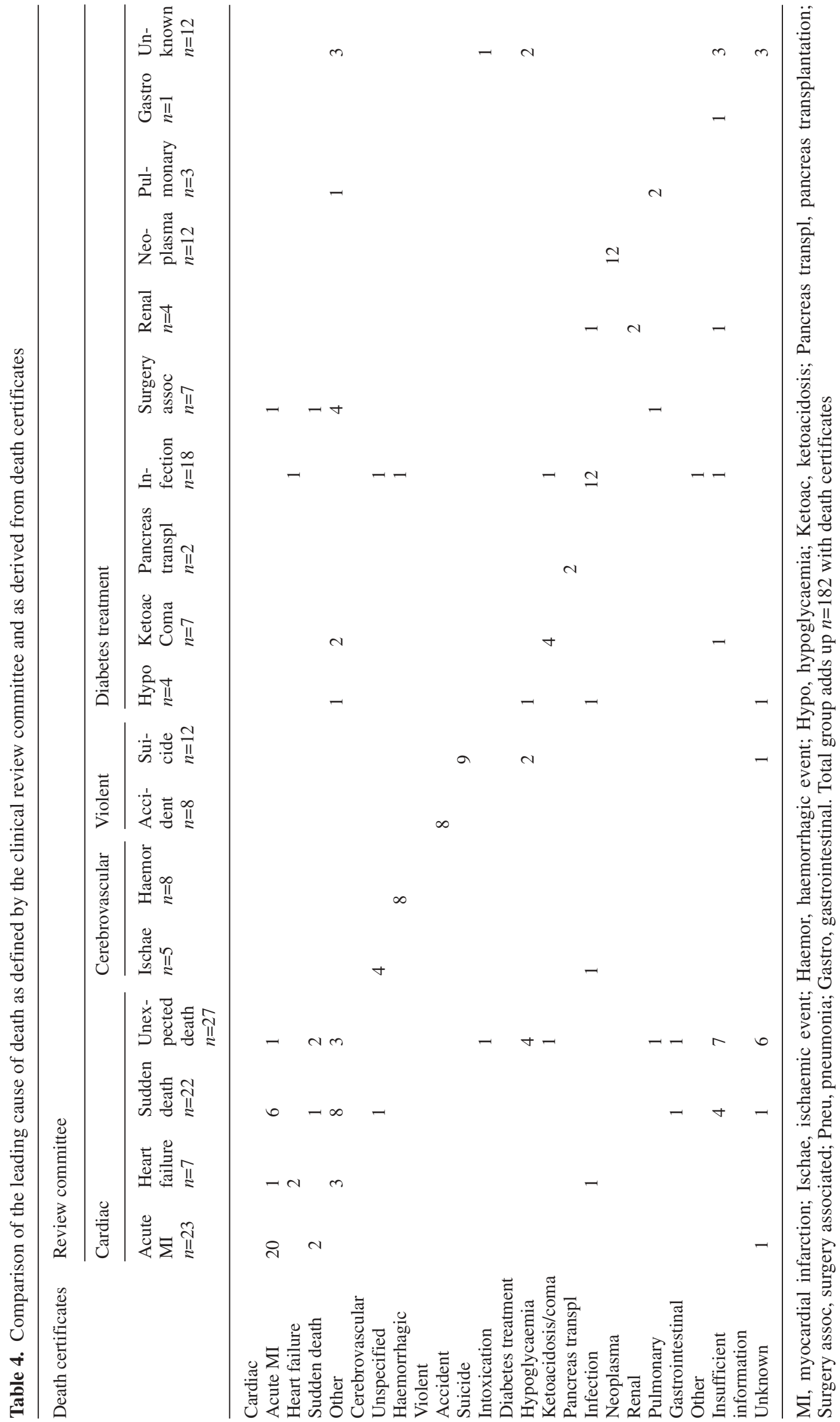




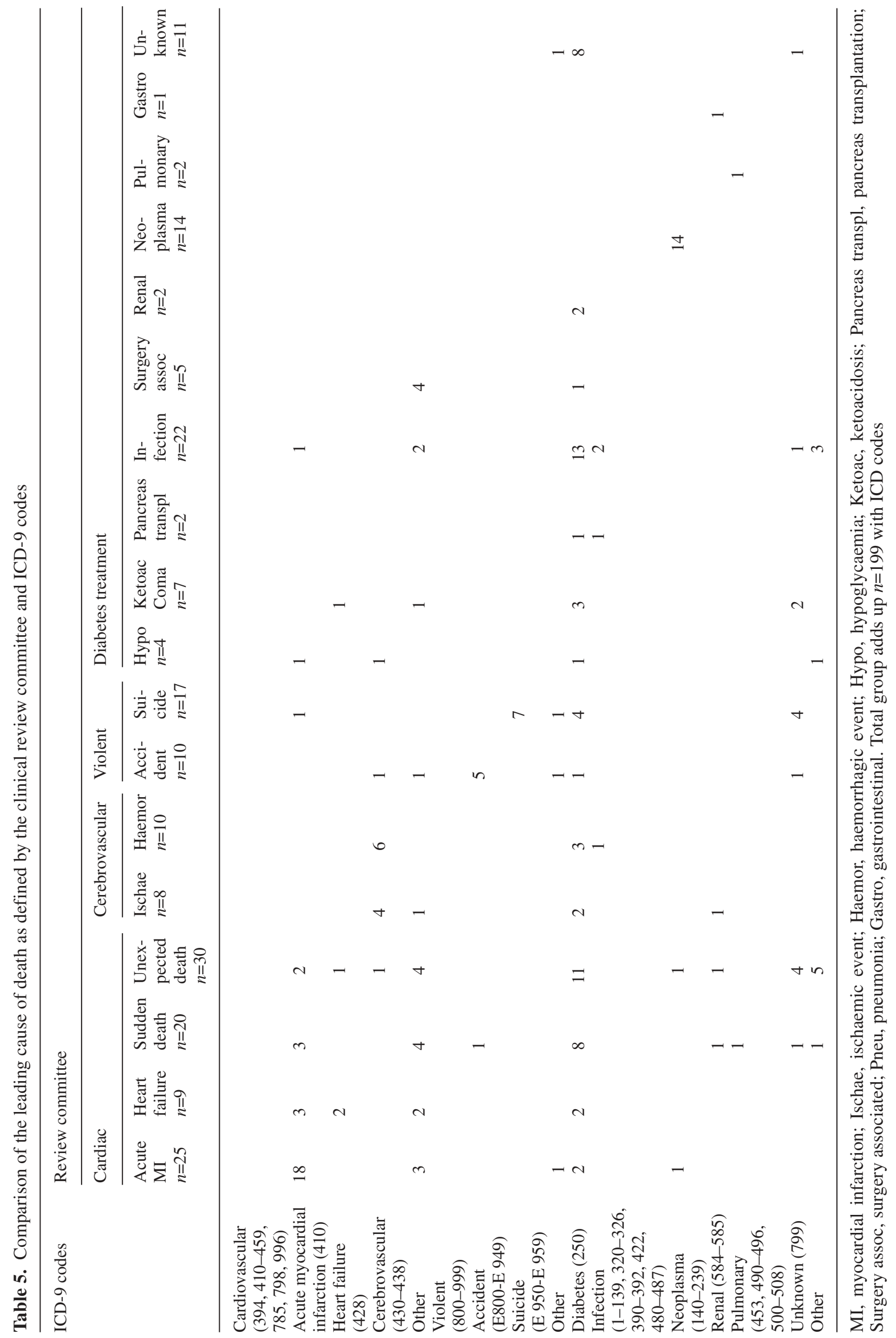


In 129 of the 182 cases $(71 \%)$ diabetes was mentioned on the death certificate. The mentioning of diabetes was particularly low in patients who had died of neoplasma or of violence. Of the 69 patients with documented renal replacement therapy, only in 52 cases $(75 \%)$ renal disease was mentioned on the death certificate (Table 3 ).

The two reviewers of the death certificates agreed on the leading cause of death in 161 of the 182 cases; agreement was achieved for the remaining cases by discussion. For $18(9.9 \%)$ death certificates insufficient information was provided to define a leading cause of death. Table 4 shows the comparison of the leading cause of death as defined by the review committee and as based on death certificates. Table 5 shows the respective data for ICD-9 codes.

Cardiovascular death. There was acceptable agreement between the leading cause of death as defined by the review committee and as derived from death certificates for acute myocardial infarction and cerebrovascular events but not for other cardiovascular deaths (Table 4). Myocardial infarction was mentioned on all but two, heart failure on all and a cerebrovascular event on all but one of the available death certificates. A cardiovascular diagnosis was mentioned on 17 of 22 available death certificates of sudden death cases, but on only 6 of 27 available death certificates of unexpected death. Most patients classified as having died of acute myocardial infarction or heart failure had a cardiovascular ICD-code, whereas patients who had died of sudden or unexpected death or a cerebrovascular event were distributed over a variety of ICD-codes (Table 5 ).

Ketoacidosis, coma. The expert review committee considered ketoacidosis as the cause of death in 8 of the 251 patients. In only 4 of 7 cases with available death certificates were ketoacidosis or (pre-)coma mentioned on the death certificate. In none of 7 cases with available ICD-codes was this cause of death identifiable through ICD-codes (Table 5).

Hypoglycaemia. The expert review committee considered hypoglycaemia as the cause of death in 7 of the 251 patients. In addition, the committee stated that in 3 of the 14 deaths due to accidents, hypoglycaemia could not be ruled out as a cause of the accident. Only in one of four cases with available death certificates was hypoglycaemia mentioned. On the other hand, hypoglycaemia was suggested as a possible leading or contributing cause of death on 8 other death certificates: in 2 of the "unknown causes of death", in 2 of the "suicide" cases and in 4 of the "unexpected death" cases. None of the hypoglycaemia causes of death was identifiable through ICD-codes (Table 5 ).

Violent death - accidents. The review committee considered 14 patients to have died due to accidents
(5 motor cycle, 7 car, 1 bicycle, 1 occupational). In all instances this cause of death was also documented on the death certificate. However, only 5 of 10 cases with available ICD-codes had received a corresponding E-code. In 3 of the 14 accident cases hypoglycaemia could not be ruled out as an important contributing cause of death. In no instance was hypoglycaemia mentioned on a death certificate.

Violent death - suicides. The review committee considered suicide as the cause of death for 22 of the 251 patients. On 9 of the 12 available death certificates suicide was explicitly mentioned. However, only 7 of the 17 cases with ICD codes were classified as suicides by E-codes (Table 5).

Infection. In 25 cases the committee considered infection as the cause of death (15 sepsis, 5 pneumonia, 5 others). Although infection or sepsis were mentioned on all available death certificates only 2 were respectively ICD-coded (Table 5 ).

Neoplasma. In 14 cases the cause of death was a neoplasma. All death certificates mentioned the respective neoplasma and all had been appropriately ICDcoded.

Unknown cause of death. For 16 patients the expert committee could not define a cause of death. In 2 of these cases hypoglycaemia and in one intoxication were suggested as a possible cause of death on the death certificates.

\section{Discussion}

This study shows that assessment of causes of death based on death certificates or on ICD-codes is unreliable in adult patients with Type I diabetes. Information on death certificates did not allow identification of about $30 \%$ of patients as people with diabetes and $25 \%$ with end-stage renal disease. About half of the patients who were considered to have died of hypoglycaemia or ketoacidosis by the review committee were differently classified on the basis of death certificates. In respect of cardiovascular causes of death, only acute myocardial infarction and cerebrovascular events were identifiable as causes of death from death certificates; other causes, sudden death in particular, could not be correctly classified. In addition, a substantial proportion of infectious causes of death could not be identified. Only deaths due to neoplasma and violence could be ascertained with acceptable accuracy from death certificates.

ICD-codes were reliable only for deaths due to neoplasma and acute myocardial infarction. A discrepancy was found for violent deaths. The review committee had identified 14 cases of accidents and 22 cases of suicide. Although, there was complete agree- 
ment between the review committee and death certificates for accidents and good agreement for suicides, only half of the accidents and only 7 out of 17 cases of suicide with available ICD-codes had received the corresponding ICD-E codes.

These results show that there could be substantial differences in the estimates of causes of death depending on whether they rely upon assignments by a review committee or a large number of certifying individuals. Based on the assumption that the review committee is more accurate, the results would have important implications. The results show that there could be substantial underestimation of suicides, accidents, acute metabolic complications and infections as causes of deaths in Type I diabetes when relying on death certificates or ICD-codes.

Necropsy is considered the gold standard for ascertainment of causes of deaths; however, autopsy rates are low. In this study only $12 \%$ of the deceased patients were known to have had an autopsy. In addition, identification of hypoglycaemia or ketoacidosis by necropsy might not be possible. Therefore, hospital records are important sources of information. Although in the United States of America [13] a high discordance rate between clinical and autopsy diagnoses has been reported for malignant neoplasms a recent study from Switzerland has shown that the frequency of major diagnostic errors in patients who died in hospital was halved over 20 years to about $14 \%$ in 1992 [14]. In our study hospital records were used by the review committee as the primary source of information in about half of the cases.

Deaths that occur outside hospital and without necropsy are more difficult to assess. In our study, detailed information on the circumstances of death as provided by relatives, physicians and police reports was obtained. Nevertheless for about $6 \%$ of patients no cause of death could be defined and a further $13.5 \%$ of deaths were classified as unexpected deaths. In the latter cases no critical event or disease was apparent at least $24 \mathrm{~h}$ before death and no other cause of death could be identified by the review committee. Thus, they were categorized as cardiovascular deaths. Nevertheless, hypoglycaemia cannot be definitely excluded as a cause of death in some of these cases and has in fact been suggested on 4 of 27 available deaths certificates. We have found a substantial underreporting of accidents and suicides by the Official State Statistics (ICD codes). Underreporting of suicides has also been found in Danish men with Type I diabetes [15].

Death certificate coding practices related to diabetes show wide differences in European countries [16] and classification systems of causes of deaths based on death certificates vary across studies on Type I diabetes. In the British Diabetic Association cohort study, the condition coded to the authors as the underlying cause of death was used for analysis, except in those cases where the cause coded as underlying was unspecified "diabetes", but the full death certificate implied either renal or cardiovascular disease as the most likely cause of death. In these cases these diseases were taken as the cause of death [3].

In the Wisconsin study all medical conditions on death certificates were coded by trained nosologists. The underlying cause of death was selected by a computer programme and the cause-specific mortality analysis was based on both the underlying cause of death and any mention of a cause on the death certificate [5].

In a Danish study on cause-specific mortality all death certificates were reviewed independently by at least two observers and the primary cause of death was recorded. If the serum creatinine concentration measured at least once a year before death was greater than $500 \mu \mathrm{mol} / \mathrm{l}$ the cause of death was coded as end-stage renal disease independent of the cause of death on the death certificate. Additional information from necropsy reports was included in cases of hypoglycaemia-related death or hyperglycaemia-related death [4].

In the WHO Multinational Study of Vascular Disease in Diabetes an independent committee of physicians assessed the information available for each patient. The information provided by the centres derived a "confidence score", with the best score being awarded if the death certificate was available together with supporting data such as a summary of terminal illness or autopsy report. The medium score was awarded if only the death certificate was available and the lowest score if the death certificate was not available but other clinical information was given [17].

In the DERI causes of death as assessed by a mortality classification committee and by death certificates were compared [9]. The study showed the inadequacy of death certificate classification alone. The application of the standardized procedure showed a major redistribution of the causes of death. The changes resulted from both the review process and the inappropriate completion of the original death certificates. Without the review, the proportion of cases due to acute complications and kidney disease would have been seriously underestimated. Furthermore, the differentiation between different diabetic causes of death could not have been done without in-depth review for each individual patient. The findings of DERI are supported by the findings of our study.

This study has several limitations which should be taken into account when interpreting the results. The analysis was retrospective and covered deaths that had occurred over a period of about 20 years. Only for patients followed in the outpatient clinic or within long-term clinical studies were circumstances and causes of death immediately investigated. A higher degree of accuracy in ascertaining causes of deaths might be achieved if a review committee would al- 
ways act shortly after a patient has died. Although a comparably high number of 251 deaths of patients with Type I diabetes could be evaluated, numbers of deaths in the various categories were small, leading to rather imprecise estimates. The generalizability of the findings of this German study is not clear. Completion of death certificates and ICD-coding could be of higher quality in other countries. In Germany, systematic quality assessment of the accuracy and coding practices for the causes of deaths is lacking. An exceptionally strict data protection system prohibits the general use and hence the critical evaluation of death certificates and ICD-codes. However, available data suggest that the validity and reliability of official mortality statistics are poor in Germany [18].

In trying to make a proposal for improved practice in defining causes of death for patients with Type I diabetes in clinical research we would suggest the following: causes of death should be assessed by a clinical review committee shortly after a patient's death; all available sources of information should be used; the limitation of information provided by death certificates should be taken into account; a leading cause of death based on consistent and written criteria should be assessed. The classification system as used by the clinical review committee in this study could be applied and further evaluated.

In conclusion, the present study underscores the need to assess causes of death in Type I diabetes by review committees. Death certificates and ICD-codes are not reliable sources of information which lead to substantial misclassification and underreporting of specific causes of deaths which are of particular relevance for Type I diabetes such as acute metabolic complications, suicides and infections.

Acknowledgements. This study has been funded by a grant of the Public Health Research Network of North-Rhine Westfalia (project II-1, no. 701400 043). We thank the Peter-Klöckner Stiftung (Duisburg) for generous financial support (grants to Prof. M. Berger).

\section{References}

1. Andresen EM, Lee JA, Pecoraro RE, Koepsell TD, Hallstrom AP, Siscovick DS (1993) Under reporting of diabetes on death certificates, King County, Washington. Am J Public Health 83: 941-943

2. Fuller JH (1993) Mortality trends and causes of death in diabetic patients. Diabetes Metab 19: 96-99
3. Laing SP, Swerdlow AJ, Slater SD et al. (1999) The British Diabetic Association Cohort Study, II: cause-specific mortality in patients with insulin-treated diabetes mellitus. Diabet Med 16: 466-471

4. Rossing P, Hougaard P, Borch-Johnsen K, Parving HH (1996) Predictors of mortality in insulin dependent diabetes: 10 year observational follow up study. BMJ 313: 779-784

5. Moss SE, Klein R, Klein BE, Meuer SM (1994) The association of glycemia and cause-specific mortality in a diabetic populaton. Arch Intern Med 154: 2473-2479

6. Lehnsten K, Becker B, Willich SN, Rjasanowski I, Michaelis D, Fischer U (1995) Mortalität bei insulinpflichtigem Diabetes mellitus in Abhängigkeit von Geschlecht, Manifestationsalter und Diabetesdauer. Diabetes \& Stoffwechsel 4: 297-303

7. Brown LJ, Scott RS, Moir CL (2001) All-cause mortality in the Canterbury (New Zealand) insulin-treated diabetic registry population. Diabetes Care 24: 56-63

8. Start RD, Bury JP, Strachan AG, Cross SS, Underwood JCE (1997) Evaluating the reliability of death in published clinical research. BMJ 314: 271

9. Diabetes Epidemiology Research International Mortality Study Group (1991) International evaluation of causespecific mortality and IDDM. Diabetes Care 14: 55-60

10. Jörgens V, Grüsser M, Bott U, Mühlhauser I, Berger M (1993) Effective and safe translation of intensified insulin therapy to general internal medicine departments. Diabetologia 36: 99-105

11. Mühlhauser I, Overmann H, Bender R, Jörgens V, Berger M (2000) Predictors of mortality and end-stage diabetic complications in persons with Type 1 diabetes on intensified insulin therapy. Diabet Med 7: 727-734

12. Mühlhauser I, Sawicki PT, Blank M, Overmann H, Bender R, Berger M (2000) Prognosis of persons with Type 1 diabetes on intensified insulin therapy in relation to nephropathy. J Internal Med 248: 333-341

13. Burton EC, Troxclair DA, Newman WP (1998) Autopsy diagnoses of malignant neoplasms. How often are clinical diagnoses incorrect? JAMA 280: 1245-1248

14. Sonderegger-Iseli K, Burger S, Muntwyler J, Salomon F (2000) Diagnostic errors in three medical eras: a necropsy study. Lancet 355: 2027-2031

15. Kyvik KO, Stenager EN, Green A, Svendsen A (1994) Suicides in men with IDDM. Diabetes Care 17: 210-212

16. Jougla E, Papoz L, Balkau B, Maguin P, Hatton F (1992) Death certificate coding practices related to diabetes in European countries - the 'EURODIAB Subarea C' Study. Int J Epidemiol 21: 343-351

17. Morrish NJ, Wang SL, Stevens LK, Fuller JH, Keen H and the WHO Multinational Study Group (2001) Mortality and causes of death in the WHO multinational study of vascular diseases in diabetes. Diabetologia 44 [Suppl 2]: S14-S21

18. Giersiepen K, Greiser E (1989) Coding of causes of death for mortality statistics - comparison of coding practices in several state statistical offices of the Federal Republic of Germany and West-Berlin. Öffentl Gesundheitswes 51: 40-47 